\title{
Omalizumab serum levels predict treatment outcomes in patients with chronic spontaneous urticaria: A three months prospective study
}

Misbah Noshela Ghazanfar ${ }^{1}$, Ewa Anna Bartko ${ }^{2}$, Nicolai Skovbjerg Arildsen ${ }^{3}$, Lars Poulsen $\mathrm{K}^{2}$, Bettina Jensen ${ }^{2}$, Christian Enevold ${ }^{4}$, Jesper Grønlund Holm ${ }^{1}$, Anders Woetmann ${ }^{3}$, Niels $\varnothing_{\mathrm{dum}}^{3}$, and Simon Francis Thomsen ${ }^{1}$

${ }^{1}$ Bispebjerg Hospital

${ }^{2}$ Gentofte Hospital

${ }^{3}$ Department of Immunology and Microbiology LEO Foundation Skin Immunology Research Center University of Copenhagen Copenhagen Denmark

${ }^{4}$ Rigshospitalet

November 3, 2021

\begin{abstract}
Aim: To examine the association between serum levels and effectiveness of omalizumab in patients with chronic spontaneous urticaria (CSU), and explore patient-specific factors associated with omalizumab pharmacokinetics. Methods: Patients with CSU, who were refractory to high-dose antihistamines and who initiated treatment with omalizumab (300 mg every four weeks) were eligible for the study. Treatment was evaluated every $4^{\text {th }}$ week during 12 weeks of treatment with urticaria activity score in the past week (UAS7) as primary outcome and urticaria control test (UCT), Chronic Urticaria Quality of Life Questionnaire (CU ${ }_{2}$ QoL) and dermatology life quality index (DLQI) as secondary outcomes. Serum drug level of omalizumab was measured before (trough level) and at day seven (peak level) after each injection. Results: A total of 23 patients were included. After 12 weeks of treatment with omalizumab, an improvement of 16.8 UAS7 points (95\% CI 10.8-22.8), p<0.001 was seen. The omalizumab trough and peak levels were $7.0-33.1 \mu \mathrm{g} / \mathrm{mL}$ and $11.4-54.0 \mu \mathrm{g} / \mathrm{mL}$ and reached a plateau (steady state) after 8-12 weeks of treatment. Among the patient-specific factors measured at baseline (age, sex, body mass index (BMI), angioedema, basophil histamine release (HR) test, blood basophils and eosinophils, and serum total IgE), BMI was the only significant predictor of omalizumab peak concentrations during the study (difference $-2.75, \mathrm{p}<0.05$ ), whereas omalizumab trough concentrations were significantly associated with UAS7 scores (difference $-0.82, \mathrm{p}<0.001$ ). The same was observed for UCT, DLQI, and CU ${ }_{2}$ QoL. Conclusion: In patients with CSU initiating treatment with omalizumab, a higher BMI predicts lower peak concentrations of omalizumab during treatment, whereas lower trough concentrations of omalizumab are associated with a poorer response on UAS7 and other patient reported outcomes.
\end{abstract}

\section{Hosted file}

serumomalizumab3.docx available at https://authorea.com/users/444333/articles/544097omalizumab-serum-levels-predict-treatment-outcomes-in-patients-with-chronic-spontaneousurticaria-a-three-months-prospective-study 